OPEN ACCESS

UWS Academic Portal

\title{
A hybrid intelligence-based cognitive engine
}

Olaleye, Martins; Dahal, Keshav; Pervez, Zeeshan

Published in:

Proceedings of the 9th International Conference On Cloud Computing, Data Science and Engineering Confluence 2019

DOI:

10.1109/CONFLUENCE.2019.8776899

Published: 29/07/2019

Document Version

Peer reviewed version

Link to publication on the UWS Academic Portal

Citation for published version (APA):

Olaleye, M., Dahal, K., \& Pervez, Z. (2019). A hybrid intelligence-based cognitive engine. In Proceedings of the 9th International Conference On Cloud Computing, Data Science and Engineering Confluence 2019 (pp. 258262). IEEE. https://doi.org/10.1109/CONFLUENCE.2019.8776899

\section{General rights}

Copyright and moral rights for the publications made accessible in the UWS Academic Portal are retained by the authors and/or other copyright owners and it is a condition of accessing publications that users recognise and abide by the legal requirements associated with these rights. 
“(C) (C) 2019 IEEE. Personal use of this material is permitted. Permission from IEEE must be obtained for all other uses, in any current or future media, including reprinting/republishing this material for advertising or promotional purposes, creating new collective works, for resale or redistribution to servers or lists, or reuse of any copyrighted component of this work in other works." Olaleye, M., Dahal, K., \& Pervez, Z. (2019). A hybrid intelligence-based cognitive engine. In Proceedings of the 9th International Conference On Cloud Computing, Data Science and Engineering Confluence 2019 (pp. 258-262). IEEE. https://doi.org/10.1109/CONFLUENCE.2019.8776899 


\section{A Hybrid Intelligence-Based Cognitive Engine}

\author{
Martins Olaleye \\ School of Computing, \\ Engineering \& Physical Sciences \\ University of the West Scotland \\ Scotland, United Kingdom \\ Martins.Olaleye@uws.ac.uk
}

\author{
Keshav Dahal \\ School of Computing, \\ Engineering \& Physical Sciences \\ University of the West Scotland \\ Scotland, United Kingdom \\ Keshav.Dahal@uws.ac.uk
}

\author{
Zeeshan Pervez \\ School of Computing, \\ Engineering \& Physical Sciences \\ University of the West Scotland \\ Scotland, United Kingdom \\ Zeeshan.Pervez@uws.ac.uk
}

\begin{abstract}
Demand for a reliable and adaptive intelligence generalization system has become an essential task to both the WCS's developers and its numerous services providers. Since WCS's spectrum is naturally known to be unstable, timedependent and currently not only scarce in capacity but heavily congested and the impacts of its various services and its rapidly evolving applications are constantly making the system to be extremely complex. However as proposed by Mitola in 1999, Cognitive Radio (CR) has been developed with such intelligence capabilities and through it, the present-day WCS's spectrum complexity can be effectively managed, at the same time increases its scarce and the highly varying spectrum utilization particularly in complicated WCS's environments. To address this, CR system through its intelligence mechanism, which is also known as the Cognitive Engine (CE) enforces such adaptive intelligence functionalities to dynamically adjust its input parameters, observing its surrounding environment and ultimately makes its decision to meet the WCS's desired objective. This paper proposes the hybridization of two different Artificial Intelligence systems to design and implement an adaptive intelligence system for CR systems to predict the WCS's required objective.
\end{abstract}

Keywords Wireless Communication System, Machine Learning, Fuzzy Logic, Random Neural Network, Neuro-fuzzy, Intelligent system hybridization, Cognitive Radio, Cognitive Engine

\section{INTRODUCTION}

One of the significant of CR system intelligence adoption in todays WCSs performances is in its ability to manage the WCS's noisy medium and its ever turbulence spectrum complexities. The spectrum is not only nature dependent, it is limited, regulated and very expensive to procure. However, a greater percentage of this resource - called spectrum, which is known to be valuable yet natural are being underutilized and in most countries of the world, most of the government regulatory bodies are being confronted with the challenges of both over-regulation and under-utilization. Hence, for the current WCS's performance to be enhanced and for the expensively allocated spectrum to be effectively utilized an efficient spectrum management scheme is now critically required [1].

Through the CR systems intelligence capability in CE, the radio is able to proffer an enhancing solution for the current WCSs users and providers to keep up with the present- day WCS's performance deterioration. The complexities status generally resulted from its continuously time-varying and dynamic environmental medium [2]. Fundamentally, CR input parameters are classified into two parts, namely environmental parameters (EV-P) and transmission parameters (TX-P). However, for the CR system to execute these highlighted functions, these two input parameters must be autonomously reconfigured, which can either be in a reactive process or proactive manner [3].

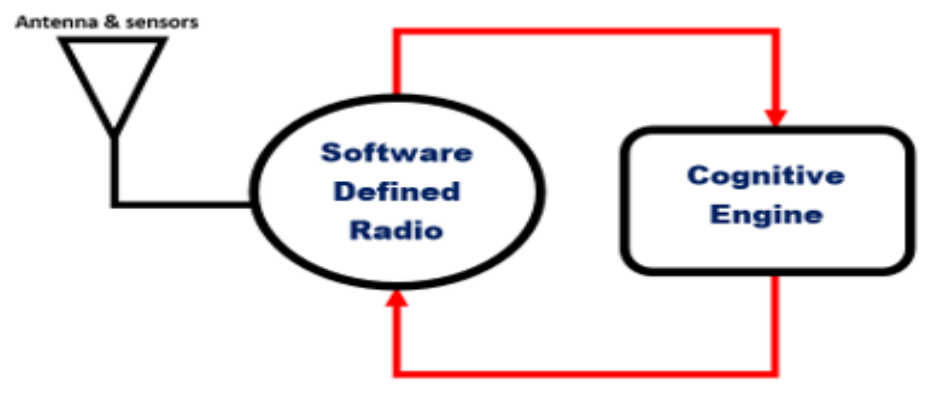

Fig. 1: The Cognitive Radio System Framework

According to Joseph Mitola [4] in the year 1999, CR system was proposed as the combination of two different softwaredeveloped units, namely Software Defined Radio (SDR) and another software-based intelligently developed package termed as cognitive engine as depicted in Fig. 1. The SDR is used to replace the conventional semi-conductor radio framework and comprises of all functional radio communication features, such as modulation, frame-size, transmit power, bandwidth, and many other features. While the $\mathrm{CE}$ is equipped with cognitive responsibilities [4], [5].

However, after Mitola proposal, Haykin in [5] was able to come up with a more advance CR system. Which was described the term CR as an intelligent-based radio that is enable with artificially developed intelligence capability to senses its operating environmental space and at the same time able to discharge its intelligence functionalities to reconfigure its operational radio parameters dynamically for it to adapt, 
learns and operates under any WCS's domain [2], [4], [5]. Based on the Haykin reviewing and as adopted by Clancy et. al in [6], CE unit was further divided into three distinct intelligence-based segments, namely as the knowledge-based engine, reasoning engine and learning engine as shown in Fig. 2.

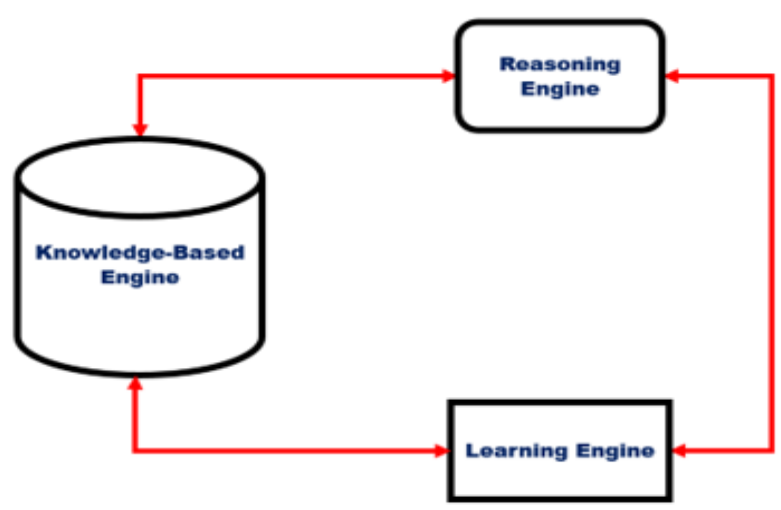

Fig. 2: Cognitive Engine Block Diagram

All the three units are essential, but out of these three, learning engine is more important. As the unit is responsible for the CR system to adapt dynamically to its surrounding environment. Such that through learning, the radio is able to develop a well-composed knowledge-based capability as well as being able to determines and enforces significant decisions in meeting the WCSs desired objective. Likewise, through the learning engine, CR systems are able to learn from the radio using its various past operations as lessons and effectively transforming it into knowledge as well as stocks them into the knowledge-based engine. From where the $\mathrm{CR}$ can easily access, most especially when requires for any future parameters reconfigurations including in making adequate decisions. While the reasoning engine over-looks the WCS's spectrum state and through it allows the radio to execute its final decision. Although reasoning engine functionality is known to be computationally intensive and timeconsuming. CR operations are in three stages, which are sensing, intelligence functionality and decision-making [2], [5], [7].

With the first stage being sensing and is the stage where through capable sensors, the WCS's surrounding environmental states are measured, e.g. interference, congestion, crosstalk, delay, tempera and more importantly its complexity status. Following this is the intelligence generalization and deployment; this is the stage where CE discharges its intelligence features to dynamically adjust all input parameters values until the radio can match or adapt to the specific condition. In this stage, learning engine also plays a vital role as knowledge is being exploited and developed into the radio and with reference to the WCS's channel estimation, WCS's resources are being predicted. Likewise, it is at this stage that every relevant past measurements, actions taken and previously exercised experiences were accounted for. Finally, the radio concluded its operation in the third stage and at this stage best input parameters configuration is obtained for the radio [8].

From the available literature in [2], [5], [7], [8], different ML techniques have been studied, proposed and applied to develop CE for CR system starting from pure lookup tables to neural networks in various learning optimization techniques, such as supervised, unsupervised and reinforcement approach, Others includes hidden Markov models, case-based, mathematically developed and evolutionary algorithms. However, by hybridizing two different machine learning techniques, this paper proposed through simulation a hybrid intelligence engine for CR systems. This was designed and implemented by arbitrarily combined the Random Neural Network-based learning algorithm as an adaptive intelligence controller with the Fuzzy Logic -type-1 prediction mechanism.

\section{Proposed Methodology}

By adopting the best of a basic Adaptive Neural Fuzzy Inference System (ANFI), which comprises of the combination of ANN with FLS. However, the proposed hybrid intelligence system as depicted in Fig. 3 was designed and implemented in this paper, by replacing the conventional ANN with a highlevel NN-based intelligence generalization algorithm using Random Neural Network-based learning algorithm.

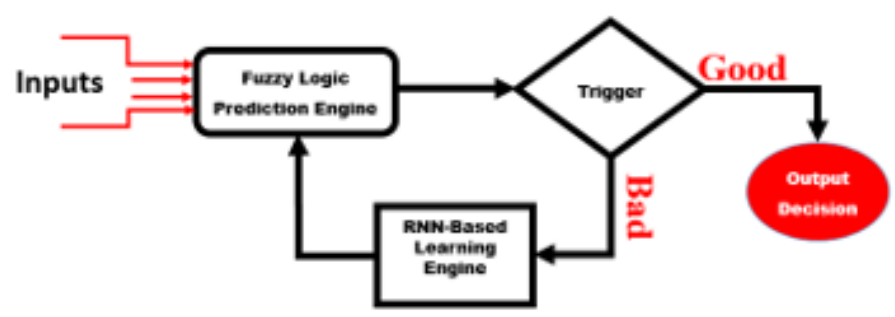

Fig. 3: The Proposed Hybrid Intelligence Model Framework

\section{A. Fuzzy Logic-Based Prediction Modelling}

Through fuzzy set theory and application developments, FLS has shown satisfactory results in modelling non-linear functions to proffer effective solutions to uncertainty, vague and complex problems. Based on this, a faster generalization prediction mechanism based on standard FL system was designed and simulated. Although, FL-based prediction algorithm has attracted significant research attention in decision making towards enhancing WCSs performance and reasonable achievements recorded in some highly cited publications such as spectrum management in [9], [10], power management in [11], optimization in [12] and natural resource management [13], [14].

According to Zadeh [15], FL is made up of four different units, namely fuzzification, inference engine and deffuzification. The FLS-based prediction model is known to fundamen- 
tally depend on the expert knowledge to formulate its inference engine. Which is made of the combination of a well-defined rule-based system (RBS) and an inference system. According to Wang and Mendel [16], who confirmed that based on human experience, defined expert or available numerical data, FL's rules can be expressed by combining Equation (1) and Equation (2) as follows:

if ... $\left(F_{\text {Noise }}=E_{i}\right) \wedge\left(U_{d}=U_{i}\right) \wedge\left(D_{r}=D_{i}\right) \wedge\left(T x_{p w r}=T_{i}\right)$

$$
\text { then } \ldots . . T_{\text {put }}=\Gamma_{(\text {Throughput })}
$$

Where:-

$$
\begin{array}{lll}
M F & = & \text { Membership Functions } \\
E_{i} & = & \text { Floor noise }-(d B) \\
U_{i} & = & \text { Nodes } \\
D_{i} & = & \text { Data rate }-(\text { Mbps }) \\
T_{i}= & \text { Transmitting power }(\text { Watts }) \\
\Gamma_{(\text {Thrō ghput }} \text { Overall Throughput }(M b)
\end{array}
$$

Logically, by expression of definition the two combined equations is known as the Rule Based System (RBS) [15], with the "IF" and "THEN" conditional factors being known as antecedent and consequences respectively. While a Shannon channel capacity equation [17] was used as the expert to formulate various rules as applied in [18]. With good implication factors of OR and AND the inference system was established. While the defuzzifier transforms the fuzzy output from the inference into real numbers and mathematically expressed in Equation (3)

$$
\text { CrispOutput }=\int \frac{\Gamma_{(\text {overall })} * \mu_{\text {out }}\left(\Gamma_{(\text {overall })}\right)}{\mu_{\text {out }}\left(\Gamma_{(\text {overall })}\right)} . \delta \Gamma_{(\text {overall })}
$$

\section{B. Random Neural Network-Based Learning Modelling}

RNN-based learning optimization paradigm was integrated with the FL-based prediction module to obtain better adaptation policy for the CR system to reconfiguring its input parameters appropriately. To design the proposed hybrid intelligence generalization in order to imitate the human brain, the process was carried-out in two respects; (1) through learning development suitable knowledge is produced and (2) through inter-connected neurons initialization, which involves weights and bias variations gets the acquired knowledge stored [3], [8]. With this, the RNN becomes a self-organized controller with accurate prediction and less dependency on any expert knowledge.

\section{1) Database Formulation and Collection: .}

The database used for both for training and testing the RNN was obtained through the design and simulation of a CR-based wireless network using the Network Simulation -2 package.
The acquired database is comprised of various CR system input parameter setting and their corresponding output result as characterize the problem to solve. By means of five input and one output parameters, the RNN model was developed in solving the problem. The input parameters used in this paper are environmental data, mobility (Nspeed), data rate (Drate), Users density (Udensity) and Transmitting power (Tpower). While the model output variable is given as the overall amount of total throughput available for the WCS within the operating domain. Although NS-2 output its results was in trace-file format and to analyse it, an Awk patched NS-2 script was employed to translate all the captured nodes communication information from the trace-file into corresponding overall throughput values for each parameter configuration. The NS-2 simulation setting are listed in Table I as follows:

TABLE I: Simulation Setting

\begin{tabular}{|l|l|}
\hline Parameters & Values \\
\hline Area of Simulation & 500 X 500 Meters \\
\hline Number of Nodes & $\begin{array}{l}\text { Minimun=50 } \\
\text { Maximum }=1000\end{array}$ \\
\hline Routing protocol & $\begin{array}{l}\text { AODV, DSDV } \\
\text { DSR and OLSR }\end{array}$ \\
\hline Mobility & 0 120 MPH \\
\hline Propagation Modes & $\begin{array}{l}\text { FreeSpace } \\
\text { Two-Ray Ground } \\
\text { Shadowing }\end{array}$ \\
\hline Antenna Model & Omni-directional \\
\hline Packet Type & TCP \\
\hline
\end{tabular}

The simulated NS-2 results were comprehensively collected to develop the database and was used both for training and testing the RNN. To meet the CR system desired prediction objective, the RNN was developed with appropriate training architecture, which includes data treatment, normalization and cross-validation. Although the data collection was through simulation and can be used just like that, yet we the decision has been made to treat the data in order to eliminate any noisy attribute. After the K-fold validation has been selected, the constructed RNN model was later trained with the dataset. In developing the RNN learning algorithm, the focus was based on how fast the learning generalization can be processed, its convergence, best parameter reconfiguration, prediction accuracy and overall optimizing the WCS's performances. Based on the simulation setting listed in Table I, in overall about 3000 data points were collected. In order to make all the data points available during the training period, K-fold data validation was used. Through this, the testing dataset was part of data points used in training the proposed RNN, while the validation was done with about 250 data points.

The developed RNN model was trained to predict the available overall throughput and was later tested with unknown testing data to validate the RNN's prediction capability. To obtain a better generalization for the RNN's input-output mapping outcome, the model trained by varying some known NN-based training factors, such as the number of neurons in the hidden layer, getting appropriate learning rate and training 


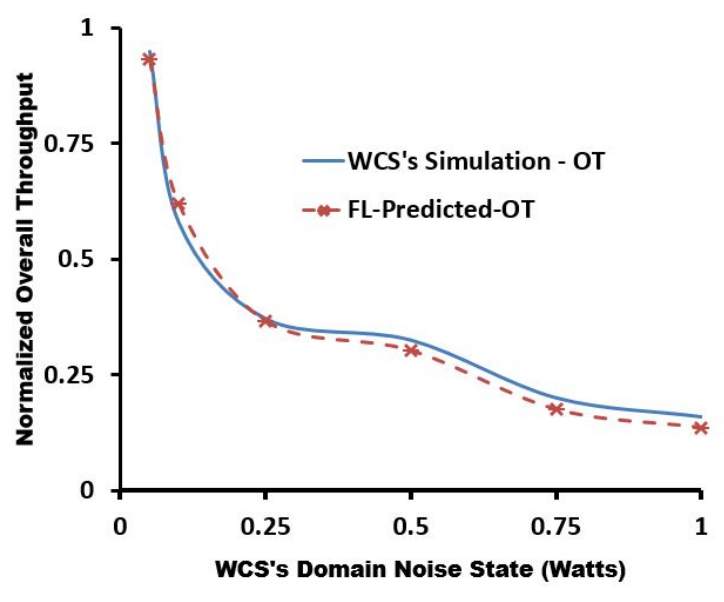

(a) FL vs NS-2 simulated output Prediction Performance

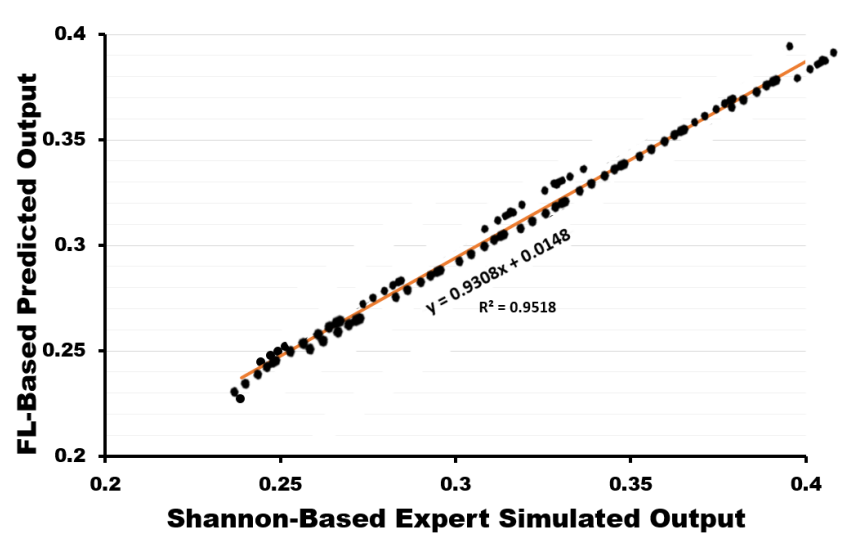

(c) Correlation Coefficient Analysis for FL Prediction

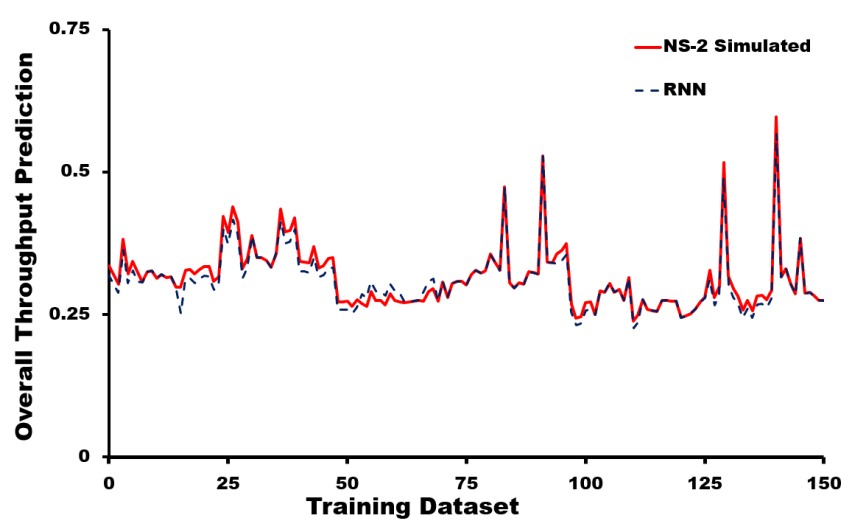

(b) RNN Training Generalization Output

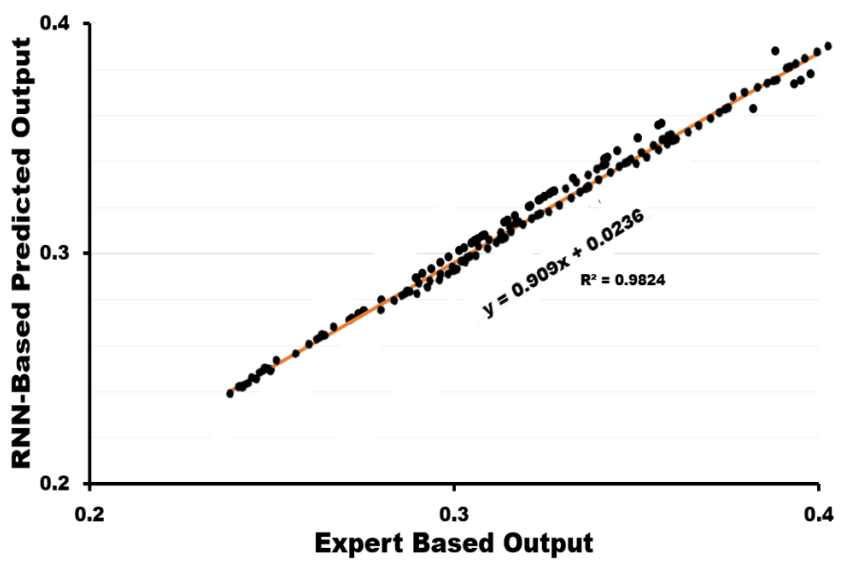

(d) Correlation Coefficient Analysis for RNN Prediction

Fig. 4: Prediction Performance Analysis for FL and RNN

epochs. Finally, few common loss-functions were selected to determine how closed the predicted outputs are to the supplied target data. Therefore, three of these commonly loss-functions were used as the trained RNN performance index were Mean Square Error (MSE), the Root Mean Square Error (RMSE) and Regression coefficients - $\left(R^{2}\right)$.

\section{RESUlt AND Discussion}

In this study, overall throughput prediction was performed using FL and RNN. The performance comparisons were evaluated between the simulated data samples and the two models predicted results. The performance comparisons between the expert-based simulated results and FL-based prediction mechanism for the available overall throughput within a given WCS's domain is presented in Fig 4a. While that of RNN is presented in Fig $4 \mathrm{~b}$ showing the loss-function margin between the training dataset versus the RNN predicted overall throughput. From Fig $4 \mathrm{c}$ and Fig $4 d$ present the closeness in terms of their linearity between the simulated dataset and the models' predicted outputs, which is the amount of overall throughput. In Fig 4c, the $R^{2}$ value of 0.9518 was obtained for the FL-Based prediction model, while 0.9824 was the $R^{2}$ value obtained from the $\mathrm{RNN}$-based learning controller as shown in Fig 4d. The obtained result from the RNN was much anticipated, since RNN model was assumed to be closer to the human intelligence generalization than the ANN and FL. Although, the selected expert for FL was equally expected to be more than this, nevertheless, Shannon channel capacity formula was generic and modelled for ideal WCS's environmental state without noise.

Finally, the two were combined together to produce the proposed hybrid neuro-fuzzy intelligence system. Thereafter, the model was subjected to a continuous simulation process, where the users are simulated for a longer distance communication and as they move they were permitted to use different services, different mobility speed and operates with different domains. In overall, the FL-based prediction mechanism was able to achieved about $89.25 \%$ predicted resources accurately above the users requirements. While about $6.14 \%$ was predicted exactly as the users demand and about $4.61 \%$ was below. Above all, the FL-based prediction controller was able to achieved all this within a very reasonable period as presented 


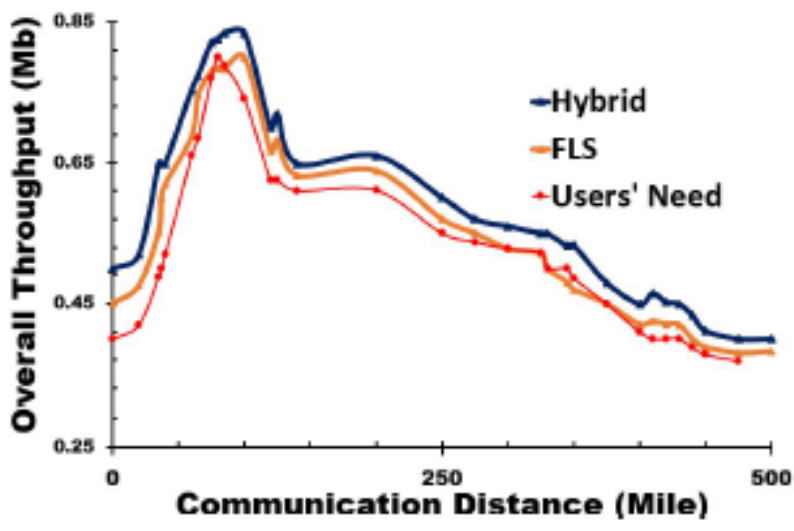

Fig. 5: Hybrid Intelligence Generalization Performance

in Table II.

TABLE II: Generalization Processing Time

\begin{tabular}{|c|c|}
\hline Model & Overall Processing Time (Seconds) \\
\hline FL & 125 \\
\hline RNN & 147 \\
\hline Hybrid & 150 \\
\hline
\end{tabular}

From Table II, it can be seen that the FL-based prediction was $16.1762 \%$ faster that the RNN and $17.518 \%$ than the hybrid. Although, RNN prediction generalization is somehow better than FL, while the different between the RNN and FL interacting with each other was as small as $1.342 \%$. With the this margin difference, this then confirmed that the hybridization triggering point for the two units has been well executed.

\section{CONClusion}

With WCS's tremendous benefits and its acceptance as well as ubiquitous to every mankind, space and time has made the system to still continues to enjoy great importance as well as exponential growth to its various services and applications development. However, with respect to the present-day WCS's environmental parameters and the desire to achieve better performance. Since the CR system's topics are diverse and there are requirements for good intelligence generalization feature for the radio's $\mathrm{CE}$, such that by efficiently reconfiguring its transmission parameters with any set of environmental parameters or WCS's environmental factors. The CR can effectively adapts or access any licensed medium yet free and overall enhances the WCS's performances. This paper has been able to present a novel neuro-fuzzy based hybrid intelligence for cognitive radio systems and with its incorporated adaptive intelligence ability, the current WCS's everdynamic, congested environmental medium and its heavy data demanding services have been effectively managed. While our future intention is to see how the proposed hybrid intelligence model can be adopted for other importance WCS's objectives, such as minimizing Bit-Error-Rate (BER), power consumption reduction and minimizing interference contribution.

\section{REFERENCES}

[1] Peter Anker, "From spectrum management to spectrum governance" Telecommunications Policy, vol 41(5), pp.486 - 497, 2017.

[2] M. A. Alsheikh and S. Lin and D. Niyato and H. P. Tan, "Machine Learning in Wireless Sensor Networks: Algorithms, Strategies, and Applications". IEEE Journal on Communications Surveys Tutorials, 2014.

[3] Wen Chen and Tao Li and Tao Yang, "Intelligent control of cognitive radio parameter adaption: Using evolutionary multi-objective algorithm based on user preference". Ad Hoc Networks, vol 26(1), pp.3 - 16, 2015.

[4] J. Mitola, "Software radios - Survey, critical evaluation and future directions". IEEE Journal on Aerospace and Electronic Systems Magazine, pp. 25-36, vol. 8,1993.

[5] Haykin, S. "Cognitive Radio: Brain-empowered Wireless Communications". Selected Areas in Communications, IEEE Journal, IEEE Press, Piscataway, NJ, USA, pp. 201-220, 2006.

[6] Clancy C, Hecker J, Stuntebeck E, OShea T.. Applications of machine learning to cognitive radio networks. IEEE transaction on Wireless Communication, pp. 4752, 2007.

[7] M. Olaleye and K. Dahal and Z. Pervez "Cognitive Radio Engine Learning Adaptation" 10th International Conference on Software, Knowledge, Information Management Applications (SKIMA), pp.325-332, Dec. 2016.

[8] K. Tsagkaris and A. Katidiotis and P. Demestichas, "Neural networkbased learning schemes for cognitive radio systems". Journal of Computer Communications, vol. 31(14), pp.3394 - 3404, 2008.

[9] P. Maheshwari and A. K. Singh, A fuzzy logic based approach to spectrum assignment in cognitive radio networks. IEEE International Advance Computing Conference (IACC), pp. 278-281, June 2015.

[10] A. Sahoo and D. D. Seth, A fuzzy logic based spectrum allocation technique for cognitive radio network. International Conference on Electrical, Electronics, Signals, Communication and Optimization (EESCO), pp. 1-4, Jan. 2015.

[11] Z. Tabakovic and S. Grgic and M. Grgic, Fuzzy Logic Power Control in Cognitive Radio. 16th International Conference on Systems, Signals and Image Processing, pp. 1-5, June 2009.

[12] N. Baldo and M. Zorzi, "Fuzzy logic for cross-layer optimization in cognitive radio networks". IEEE Communications Magazine, vol. 46, no. 4, pp. 64-71, April 2008.

[13] Yeon Kwan Kang and Hyeonmin Kim and Gyunyoung Heo and Seok Yoon Song, Diagnosis of feedwater heater performance degradation using fuzzy inference system. Expert Systems with Applications, Vol.69, pp. 239-246, 2017.

[14] Boyacioglu, Melek Acar and Avci, Derya "An Adaptive Network-Based Fuzzy Inference System (ANFIS) for the Prediction of Stock Market Return: The Case of the Istanbul Stock Exchange". Expert System Application, vol 37(12), pp. 7908-7912, 2010.

[15] Klir, George J. and Yuan, Bo. "Fuzzy Sets, Fuzzy Logic, and Fuzzy Systems: Selected Papers by Lotfi A. Zadeh" World Scientific Publishing Co., Inc., River Edge, NJ, USA, 1996.

[16] L. X Wang and Jerry M. Mendel "Generating fuzzy rules by learning from examples". IEEE Transactions on Systems, Man,and Cybernetics, vol 22(6), pp. 14141427, 1992.

[17] C. E. Shannon, "A Mathematical Theory of Communication", Bell System Technology Journal, Vol. 27, pp.379-423, October, 1948.

[18] Tsangaratos, P.; Ilia, I., "Combining fuzzy logic and information theory for producing a landslide susceptibility model". In Proceedings of the 14th International Congress, Thessaloniki, Greece, vol. 50, pp.3446, 2016. 